\title{
Solvation in pure and mixed solvents: Some recent developments*
}

\author{
Omar A. El Seoud $\ddagger$ \\ Instituto de Química, Universidade de São Paulo, C.P. 26077, 05513-970, \\ São Paulo, S.P., Brazil
}

\begin{abstract}
The effect of solvents on the spectra, absorption, or emission of substances is called solvatochromism; it is due to solute/solvent nonspecific and specific interactions, including dipole/dipole, dipole-induced/dipole, dispersion interactions, and hydrogen bonding. Thermo-solvatochromism refers to the effect of temperature on solvatochromism. The molecular structure of certain substances, polarity probes, make them particularly sensitive to these interactions; their solutions in different solvents have distinct and vivid colors. The study of both phenomena sheds light on the relative importance of the solvation mechanisms. This account focuses on recent developments in solvation in pure and binary solvent mixtures. The former has been quantitatively analyzed in terms of a multiparameter equation, modified to include the lipophilicity of the solvent. Solvation in binary solvent mixtures is complex because of the phenomenon of "preferential solvation" of the probe by one component of the mixture. A recently introduced solvent exchange model allows calculation of the composition of the probe solvation shell, relative to that of bulk medium. This model is based on the presence of the organic solvent (S), water (W), and a 1:1 hydrogen-bonded species $(\mathrm{S}-\mathrm{W})$. Solvation by the latter is more efficient than by its precursor solvents, due to probe/solvent hydrogen-bonding and hydrophobic interactions. Dimethylsulfoxide (DMSO) is an exception, because the strong DMSO/W interactions probably deactivate the latter species toward solvation. The relevance of the results obtained to kinetics of reactions is briefly discussed by addressing temperature-induced desolvation of the species involved (reactants and activated complexes) and the complex dependence of kinetic data (observed rate constants and activation parameters) in binary solvent mixtures on medium composition.
\end{abstract}

Keywords: solvation; solvatochromism; polarity indicators; thermo-solvatochromism; binary solvent mixtures.

\section{INTRODUCTION}

The impetus for the continued interest in studying solvation is that most reactions are carried out in solution, including pure solvents and their mixtures. A literature survey in Chemical Abstracts resulted in 3392 entries containing the two concepts "reaction rate constants" and "solution" closely associated with one another, including 94 patents; the corresponding figures for the concepts "reaction equilibrium constants" and "solution" are 1216 and 6, for total entries and patents, respectively. Our interest in understanding solvation has intensified because the "green" approach to chemistry is becoming increas-

\footnotetext{
*Paper based on a presentation at the $18^{\text {th }}$ International Conference on Physical Organic Chemistry (ICPOC-18), 20-25 August 2006, Warsaw, Poland. Other presentations are published in this issue, pp. 955-1151.

‡E-mail: elseoud@iq.usp.br
} 
ingly important. The latter sets guidelines designed to secure sustainable development, while increasing process biocompatibility and economy, with emphasis on an increase in, and/or upgrading of [1-3]:

- Process economy: by preventing waste generation. This is a much superior approach to waste treatment.

- Atom economy: by incorporating all reagents employed in the final product. This also contributes to reduction and/or elimination of waste.

- $\quad$ Process safety: by using nontoxic, noninflammable solvents and reagents.

- $\quad$ Process efficiency: by material recycling into the process; use of catalytic pathways; use of catalysts that can be regenerated and/or recycled; rational use of energy; and reduction of the number of intermediate steps.

These principles call for a thorough understanding of the roles of all components of the chemical reaction/process including, naturally, the solvent or solvent mixture employed. The safety factor calls for a careful selection of the solvent; use of aromatic and halogenated solvents has noticeably decreased in organic synthesis and industrial chemical processes. Introduction of the so-called "green" solvents (of which supercritical $\mathrm{CO}_{2}$ [4] and room-temperature ionic liquids [5-7] are promising examples) has increased our need to probe solute/solvent interactions at the molecular level. The importance of understanding solvation of organic and inorganic species, as well as solvent effects on chemical phenomena, is shown by the fact that there are several books and review articles devoted to these subjects [8-16].

This article is intended to give a simplified, nonmathematical account of solvation in pure solvents and their binary mixtures. The emphasis is on the relationship between solvation and the physicochemical properties of both solute and solvent, and the effect of temperature on solvation, as deduced from our recent results. It is hoped that this review serves as a summarized introduction to solvation and helps those who need to choose a solvent, or a binary solvent mixture, for a particular application, but who do not wish to spend too much time in order to understand fully the different aspects of solvation (e.g., energetics, models, etc.).

\section{IMPORTANCE OF UNDERSTANDING SOLVATION}

The following representative examples underscore the need to understand solvation for a rational use of solvents and their mixtures (e.g., in controlling the rates and equilibrium constants of chemical reactions): On transfer from water to methanol, $\mathrm{MeOH}$, the first and second $\mathrm{p} K_{\mathrm{a}}$ of fumaric acid increase by 5.8 and 5.5 units, respectively [17]; Transfer of malachite green from bulk water to water-in-oil microemulsion formed by a non-ionic surfactant [polyoxyethylene(6)nonylphenyl ether] increases its apparent $\mathrm{p} K_{\mathrm{a}}$ by 7 units [18]; The equilibrium constant of the keto-enol tautomerism of acetylacetone is 183 times greater in cyclohexane than in water [19]; At $30^{\circ} \mathrm{C}$, ethylation of sodio butyrophenone is $75 \%$ complete in $152 \mathrm{~s}$ and $237 \mathrm{~h}$ in diethylene glycol and diethyl ether, respectively [20]; The rate constant of the $\mathrm{S}_{\mathrm{N}} 1$ solvolysis of $t$-butyl chloride in water is 335000 times larger than that in ethanol [21]; The rate constant of the $\mathrm{S}_{\mathrm{N}} 2$ iodide exchange reaction $\left(\mathrm{I}^{-*}+\mathrm{CH}_{3} \mathrm{I} \rightarrow \mathrm{CH}_{3} \mathrm{I}^{*}+\mathrm{I}^{-}\right)$is 1300 times greater in acetone than in water [22]; The rate constant of the $\mathrm{pH}$-independent hydrolysis of 4-methoxyphenyl dichloroacetate in water is 500 times greater than that in non-ionic micellar solution [polyoxyethylene(7)dodecyl ether] [23]; The half-life of the spontaneous decarboxylation of 6-nitro-3carboxybenzoxazole, Fig. 1 is 1 day in water and $0.001 \mathrm{~s}$ in hexamethyl phosphoric acid triamide [24]; Depending on the molecular structures of the diene-dieonophile pair, the Diels-Alder reaction in water is $10^{4}$ faster than that in organic solvents [25]; The energetics of reactions involving a neutral molecule and a strongly solvated species, in particular, the alkoxide and hydroxide ions, may be partially, or completely dominated by the desolvation barrier of the base, with concomitant introduction of the neutral reagent into its solvation shell [26]. 


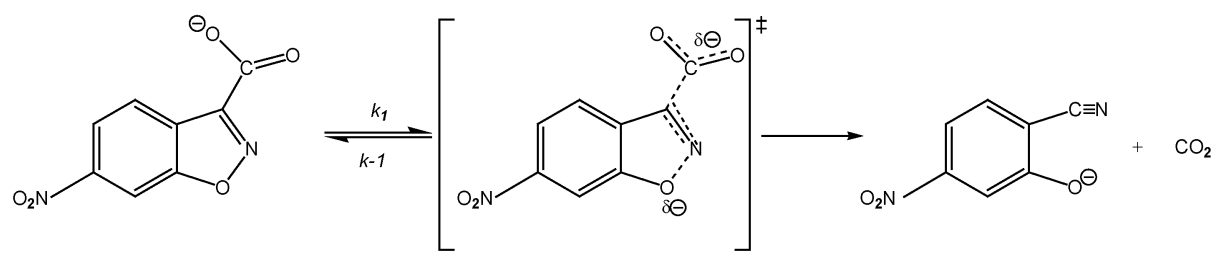

Fig. 1 Schematic representation of the decarboxylation of 6-nitro-3-carboxybenzoxazole [24].

Medium effects on reactivity are not restricted to pure solvents; some peculiar results in binary solvent mixtures underscore the need for a clearer understanding of both solvent/solvent and solute/solvent interactions. An illustrative example is the dependence of kinetic data of reactions carried out in aqueous media on $\chi_{\mathrm{w}}$ or [W] ( $\chi$ and $\mathrm{W}$ refer to mole fraction and water, respectively). These data include observed rate constants, $k_{\text {obs }}$; kinetic orders with respect to water (n), and the activation parameters. Thus, addition of water $\left(<\chi_{\mathrm{w}}=0.2\right)$, strongly inhibits the $\mathrm{S}_{\mathrm{N}} 2$ reaction between sodium 4-nitrophenolate and $\mathrm{CH}_{3} \mathrm{I}$ in aqueous acetone, followed by a much less variation at higher $\chi_{\mathrm{w}}$, i.e., at higher medium polarity [27]; Whereas the value of $(n)$ for the reversible hydration of chloral in aqueous acetonitrile, MeCN, decreases from ca. 4 to ca. 2 when [W] is increased from 0.25 to $46.3 \mathrm{~mol} / \mathrm{L}$, respectively [28], that for the $\mathrm{pH}$-independent hydrolysis of 2,4-dinitrophenyl carbonate in aqueous $\mathrm{MeCN}$ increases from 1.9 to 4.1 , when $\chi_{\mathrm{w}}$ is increased from the $0.23-0.43$ range to the $0.87-0.97$ range, respectively [29]; The number of water molecules in the TS of the imidazole-catalyzed hydrolysis of $\mathrm{N}$-(4-nitrophenyl)trifluoroacetamide in $\mathrm{W}-\mathrm{MeCN}$ was reported to increase from one to four as a function of increasing [W] [30]; For many $\mathrm{pH}$-independent reactions, the plots of $T \Delta S^{\neq}$and $\Delta H^{\neq} \mathrm{vs}$. [W] are complex, frequently showing quasi mirror-image dependences [25,29,31,32].

The dependence of $(n)$ on [W] in these aqueous media merits a comment because it underlines the need to understand solvation. For example, it is not possible to calculate $(n)$ when $k_{\text {obs }}$ decreases as a function of increasing [W]; The decrease of $(n)$ as a function of increasing [W] is counterintuitive; For a typical bimolecular reaction (e.g., ester plus water), the increase of $(n)$ as a function of increasing [W] implies that the gain in $\Delta H^{\neq}$outweighs the loss in the $T \Delta S^{\neq}$term, this is not always the case. An alternative explanation is that $(n)$ reflects not only the number of water molecules in the transition state, but also the susceptibility of the reaction to the microstructure of the medium, vide infra. Indeed, application of the proton inventory technique in order to calculate the number of hydrogens (hence, water molecules) that participate in the transition state of several $\mathrm{pH}$-independent hydrolyzes has shown that this number is constant, i.e., is independent of medium composition [31,32].

\section{QUANTITATIVE CORRELATIONS BETWEEN SOLVENT PROPERTIES AND CHEMICAL PHENOMENA}

The next question concerns the correlation between the properties of solvents, or binary solvent mixtures and their effects on rates and/or equilibrium constants of chemical reactions. It has long been realized that correlation with a single solvent property is observed only under special conditions, i.e., it is the exception, not the rule. Whereas $\log k_{\mathrm{obs}}$ for the Menschutkin reaction between triethylamine and iodoethane is linearly correlated with Kirkwood dielectric function $\left[\left(\varepsilon_{\mathrm{r} \text {,mixture }}-1\right) /\left(2 \varepsilon_{\mathrm{r}, \text { mixture }}+1\right)\right.$ where $\varepsilon_{\mathrm{r}}$ is the relative permittivity] of mixtures of acetone and 1,4-dioxane or of acetone and benzene, it is not correlated with the same function in 32 aprotic and dipolar aprotic solvents [33]. Figure 2 shows that there is no correlation between $k_{\text {obs }}$ for the spontaneous decarboxylation of 6-nitro-3-carboxybenzoxazole and $\varepsilon_{\mathrm{r}}$, a representative solvent property [24]. 


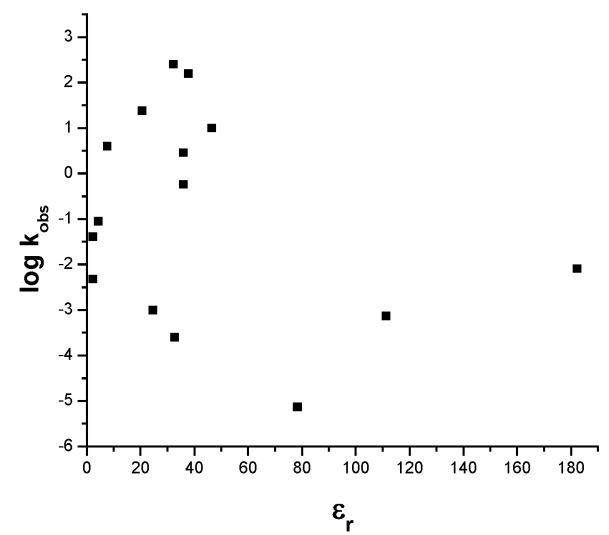

Fig. 2 Correlation between $\log k_{\mathrm{obs}}$ and $\varepsilon_{\mathrm{r}}$ for the decarboxylation of 6-nitro-3-carboxybenzoxazole in different solvents.

\section{USE OF SOLVATOCHROMIC DYES TO PROBE SOLVATION}

The data plotted in Fig. 2 neatly demonstrate that the influence of solvents on rate and equilibrium constants of chemical reactions are complex and can only be satisfactorily described by a combination of solvent properties, rather than a single one. A detailed account of the relevant solute/solvent interactions, e.g., ion/dipole, dipole/dipole, dipole-induced/dipole, and dispersion or London interactions, or of multiple correlations between chemical phenomena and solvent properties is outside the scope of this review [13]. The present discussion is limited to a single approach, based on the Taft-Kamlet-Abboud equation, which takes the form [34]:

$$
\mathrm{SDP}=\text { constant }+s\left(\pi_{\mathrm{S}}^{*}+\mathrm{d} \delta\right)+a \alpha_{\mathrm{S}}+b \beta_{\mathrm{S}}+h\left(\delta_{\mathrm{H}}^{2}\right)
$$

where the solvent-dependent phenomenon, SDP, such as rate constant, equilibrium constant, spectroscopic shift, is modeled as a linear combination of a dipolarity/polarizability term $\left[s\left(\pi^{*}{ }_{S}+\mathrm{d} \delta\right)\right]$, two hydrogen-bonding terms, in which the solvent is the hydrogen-bond donor $\left(a \alpha_{S}\right)$, or the hydrogen-bond acceptor $\left(b \beta_{\mathrm{S}}\right)$, and a cavity term $\left[h\left(\delta^{2}{ }_{\mathrm{H}}\right)\right]$, related to Hildebrand solubility parameter. The parameters $\pi_{\mathrm{S}}^{*}, \alpha_{\mathrm{S}}$, and $\beta_{\mathrm{S}}$, are known as solvatochromic parameters because they are determined by using solvatochromic probes (vide infra); the subscript ( $\mathrm{S}$, for solvent) is employed so that they are not confused with other known quantities, e.g., $\alpha$ and $\beta$ of the Brønsted equation.

Of particular interest to the present discussion is the effect of solvent on the spectra of the socalled "solvatochromic" probes, substances whose spectra, absorption, or emission are particularly sensitive to specific solvent properties ("acidity", "basicity", etc.). The information about solvation is derived as follows: From the spectra of the probe in a series of solvents, an empirical solvent polarity scale, $E_{\mathrm{T}}$ (probe) is calculated from eq. 2 :

$$
E_{\mathrm{T}} \text { (probe), } \mathrm{kcal} / \mathrm{mol}=28591.5 / \lambda_{\max }(\mathrm{nm})
$$

This equation converts the electronic transition within the probe into the corresponding intramolecular charge-transfer energy. Values of $E_{\mathrm{T}}$ (probe) are then rationalized in terms of non-specific and specific probe/solvent interaction mechanisms, vide supra. Solvatochromic data of these probes in binary solvent mixtures can be manipulated in order to determine the composition of the probe solvation shell (hereafter designated as "solvation shell"); comparison with the composition of the bulk mixture shows whether the probe is preferentially solvated by one of its components. Finally, the data of thermosolvatochromism (i.e., dependence of solvatochromism on temperature) show how solvation is affected by temperature. 
The molecular structures of the zwitterionic probes that we have employed, along with the $\mathrm{p} K_{\mathrm{a}}$ of their conjugate acids in water, $\log P$ (a measure of lipophilicity or hydrophobic character; $\log P=\log$ ([substance $\left.]_{n \text {-Octanol }} /[\text { substance }]_{\text {Water }}[35]\right)$ and the acronym of the corresponding empirical solvent polarity scales are depicted in Fig. 3 [36-45]. Historically, the solvent polarity scales of RB and WB have been termed $E_{\mathrm{T}}(30)$ and $E_{\mathrm{T}}(33)$, respectively. For simplicity, the polarity scales of other probes are designated by the probe acronym itself $E_{\mathrm{T}}(\mathrm{QB}), E_{\mathrm{T}}(\mathrm{MePM})$, etc. One common feature of these probes is that their excitation from the ground state to the first excited state is accompanied by a large change in their dipole moment, so that they exhibit distinct colors in different solvents. For example, BuQMBr ${ }_{2}$ is pink, blue, blue-ish green, and green in aqueous methanol, pure methanol, acetone, and chlorobenzene, respectively.

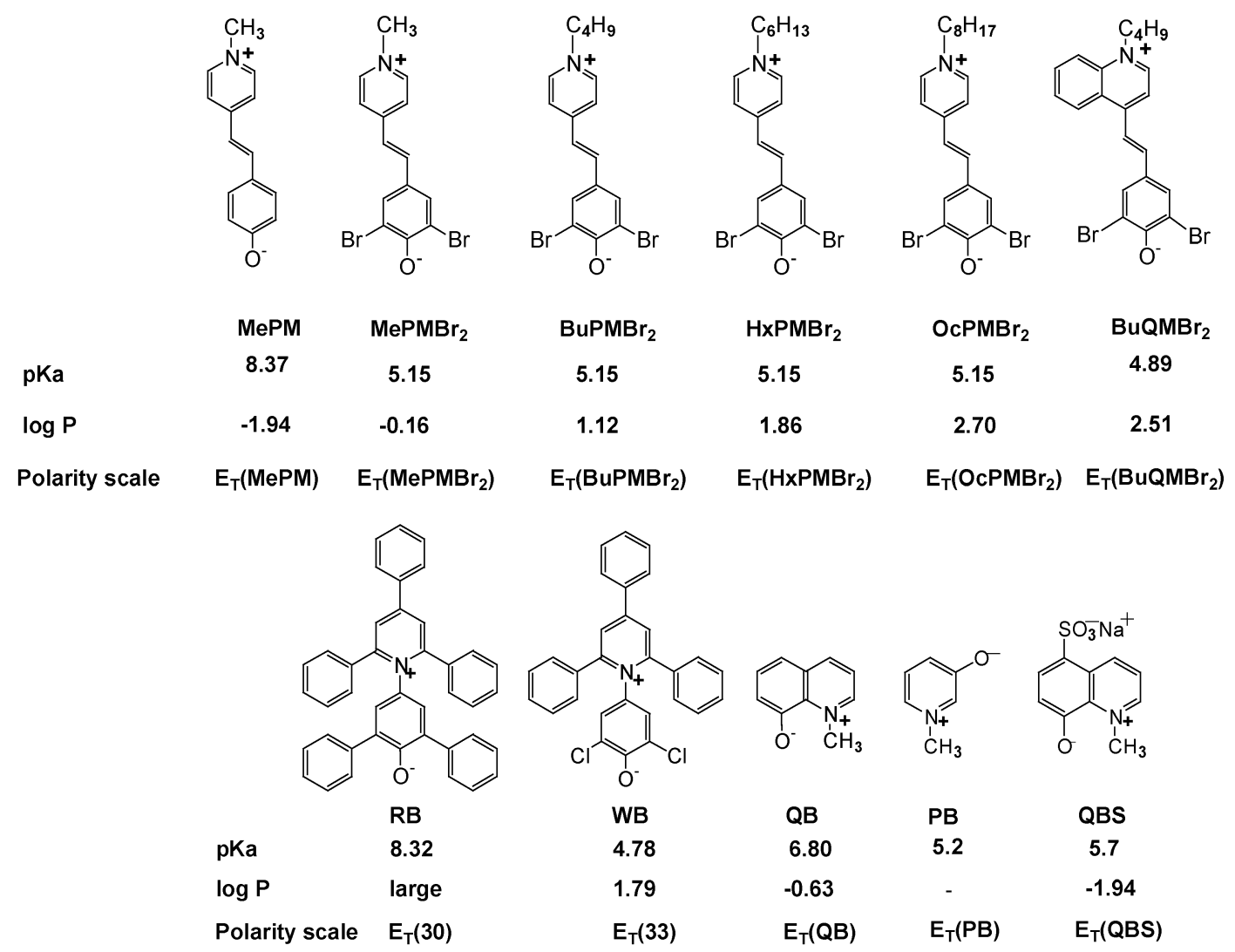

Fig. 3 Molecular structures of zwitterionic solvatochromic indicators, employed by the author, along with the $\mathrm{p} K_{\mathrm{a}}$ of their conjugate acids in water, $\log P$ (see definition in text) and the acronym for their empirical polarity scale.

\section{SOLVATION IN PURE SOLVENTS: CONTRIBUTION OF SOLVENT LIPOPHILICITY}

Since excitation of a solvatochromic probe does not involve changes in the cavity volume within the solvent, the $\left[\mathrm{h}\left(\delta_{\mathrm{H}}^{2}\right)\right]$ term is dropped and eq. 1, for a single solvatochromic probe in a series of solvents, is reduced to:

$$
E_{\mathrm{T}}(\text { probe })_{\mathrm{obs}}=\text { constant }+s\left(\pi_{\mathrm{S}}^{*}+\mathrm{d} \delta\right)+a \alpha_{\mathrm{S}}+b \beta_{\mathrm{S}}
$$

where the information required (relative contributions of solvent properties to solvation) can be extracted from the regression coefficients $(s),(a)$, and $(b)$, respectively. These coefficients permit a comparison of the response of different probes to the same solvent property, e.g., acidity or basicity. They 
do not permit, however, a direct comparison of the relative importance of solvent properties to the solvation of different probes. The reason is that $E_{\mathrm{T}}$ (probe) and the properties employed ( $\pi_{\mathrm{S}}{ }^{*}, \alpha_{\mathrm{S}}$, and $\beta_{\mathrm{S}}$ ) have different scales. Use of the corresponding standardized coefficients, $\beta_{\text {stat }}$ of (regression coefficient of solvent property), however, solves this problem [46].

What is required, therefore, is to apply multiparameter equations (e.g., eq. 3) to the solvatochromic data of several indicators in a series of solvents, in order to find out if there is a trend. A problem with this approach is that MePM, RB, WB, PB, and QB differ widely in their molecular structures, hence in physicochemical properties that are relevant to their solvation. Consequently, quantification of the effects of a single property on solvation (e.g., probe $\mathrm{p} K_{\mathrm{a}}$ or dipole moment) is not feasible because these properties change simultaneously, for some of the probes depicted. For example, although RB is much more basic than WB, the response of both probes to solvent "acidity" or hydrogen-bond donation is similar. Briefly, whereas the ability of solvents to form hydrogen bonds with the phenolate oxygen of $\mathrm{RB}$ is attenuated due to steric hindrance by the two ortho phenyl rings, the corresponding ability of WB is enhanced because of lower steric hindrance around the phenolate oxygen, and the additional ability of two ortho chlorine atoms to form hydrogen bonds [39]. Additionally, our solvatochromic data have indicated that lipophilicity of both solvent and probe are important to solvatochromism [36,38,40-42]; there is no provision for the latter property in eq. 3. This raises a question whether the latter equation should also include a term for solvent lipophilicty, as shown in eq. 4 :

$$
E_{\mathrm{T}}(\text { probe })_{\mathrm{obs}}=\text { constant }+s\left(\pi_{\mathrm{S}}^{*}+\mathrm{d} \delta\right)+a \alpha_{\mathrm{S}}+b \beta_{\mathrm{S}}+p \log \left(P_{\mathrm{S}}\right)
$$

where $\log \left(P_{\mathrm{S}}\right)$ refers to the partition coefficient of the solvent between $n$-octanol and water. In order to address both problems, we have synthesized the merocyanine series $\mathrm{RPMBr}_{2}$, where $\mathrm{R}=$ methyl to $n$-octyl. As expected, $\mathrm{p} K_{\mathrm{a}}$ values of their conjugate acids in water were found to be identical, so that they differ only in lipophilicity. These probes were employed to measure the polarity of 36 protic and aprotic solvents. Table 1 shows the results of the application of eq. 4 to the solvatochromic data of the probes depicted in Fig. 3 [45].

Table 1 Values of $\beta_{\text {stat }}$ coefficients of eq. 4 , calculated from solvatochromic data $\left(25^{\circ} \mathrm{C}\right)$ of the probes depicted in Fig. 3. ${ }^{\mathrm{a}}$

\begin{tabular}{lccrrrr}
\hline Probe & $\beta_{\text {stat }}(s)$ & $\beta_{\text {stat }}(a)$ & $\beta_{\text {stat }}(b)$ & $\beta_{\text {stat }}(p)$ & $r^{2, \mathrm{~b}}$ & $\mathrm{~N}^{\mathrm{b}}$ \\
\hline $\mathrm{MePM}_{\mathrm{MePMBr}_{2}}$ & $0.43( \pm 0.09)$ & $0.92( \pm 0.08)$ & $-0.06( \pm 0.08)$ & $-0.15( \pm 0.07)$ & 0.9542 & 36 \\
BuPMBr$_{2}$ & $0.46( \pm 0.07)$ & $0.81( \pm 0.06)$ & $0.02( \pm 0.07)$ & $-0.24( \pm 0.07)$ & 0.9258 & 36 \\
OcPMBr $_{2}$ & $0.43( \pm 0.07)$ & $0.80( \pm 0.06)$ & $-0.01( \pm 0.07)$ & $-0.21( \pm 0.07)$ & 0.9334 & 35 \\
BuQMBr $_{2}$ & $0.28( \pm 0.06)$ & $0.79( \pm 0.06)$ & $-0.01( \pm 0.07)$ & $-0.22( \pm 0.07)$ & 0.9317 & 35 \\
RB & $0.47( \pm 0.09)$ & $0.80( \pm 0.07)$ & $-0.23( \pm 0.09)$ & $-0.35( \pm 0.08)$ & 0.9011 & 36 \\
WB & $0.37( \pm 0.07)$ & $0.80( \pm 0.06)$ & $-0.02( \pm 0.09)$ & $-0.08( \pm 0.08)$ & 0.9412 & 25 \\
QB & $0.29( \pm 0.07)$ & $0.85( \pm 0.06)$ & $0.01( \pm 0.07)$ & $-0.02( \pm 0.10)$ & 0.9415 & 24 \\
QBS & $0.46( \pm 0.17)$ & $1.04( \pm 0.15)$ & $0.04( \pm 0.18)$ & $0.07( \pm 0.13)$ & 0.9088 & 17 \\
PB & $0.24( \pm 0.06)$ & $0.91( \pm 0.06)$ & $-0.11( \pm 0.06)$ & $0.05( \pm 0.09)$ & 0.9597 & 24 \\
\hline
\end{tabular}

aFrom ref. [45]. The data for QBS and PB were calculated from ref. [36].

${ }^{\mathrm{b}}$ The abbreviations $r^{2}$ and $(\mathrm{N})$ refer to the coefficient of multilinear correlation and number of solvents tested, respectively. The relatively small value of $(\mathrm{N})$ for QBS is dictated by its limited solubility in organic solvents [36].

Table 1 shows a clear trend: Probe/solvent interactions are dominated by a blend of nonspecific interactions, included in the dipolarity/polarizability term $\left(\pi_{\mathrm{S}}^{*}\right)$, and specific interactions (in particular, hydrogen bonding to the probe phenolate oxygen) included in the $\alpha_{S}$ term. Values of $\beta_{\text {stat }}$ of $\left(a_{S}\right) / \beta_{\text {stat }}$ of (s) range from 1.70 to 3.79, showing that specific interactions dominate the solvation of these zwitterionic probes. Although the scatter in the coefficients $\beta_{\text {stat }}$ of $(b)$ and $\beta_{\text {stat }}$ of $(p)$ is large for some probes, their solvation is clearly more sensitive to solvent lipophilicity than its basicity. An exception is 
PB probably because it is a very hydrophilic probe, being insensitive to solvent lipophilicity. Unlike other probes tested, the UV-vis spectrum of PB is not affected by ionic surfactants, because it does not partition into the micelle, due to its hydrophilicity [47]. For RB, the negligible dependence of $E_{\mathrm{T}}(30)$ on $\beta_{\text {stat }}$ of $(b)$ has been attributed to steric hindrance to electron-pair donation by the solvent to the heterocyclic quaternary nitrogen, due to the ortho phenyl groups [14]. The latter mechanism is not operative, however, for the merocyanine probes, because the heterocyclic rings carry no substituents in the ortho positions. However, several pieces of evidence show that the interactions between the solvent (as electron donor) and positively charged nitrogens are inefficient. For example, whereas the $\left(\mathrm{CH}_{3}\right)_{4} \mathrm{~N}^{+}$ ion has no effect on the structure of water, $\left(n-\mathrm{C}_{4} \mathrm{H}_{9}\right)_{4} \mathrm{~N}^{+}$has a net structure-enhancing effect, due to hydrophobic hydration of the alkyl groups [48]. On the other hand, addition of $\beta_{\mathrm{S}}$ to the equation that describes the dependence of Gibbs free energies of solution of tetra-alkylammonium halides on solvent properties did not increase the overall correlation coefficient, so that $\beta_{\mathrm{S}}$ was dropped [49]. We have tested eq. 4 for a total of 23 solvatochromic probes of different molecular structures, where adequate numbers of solvents have been tested. The same conclusion about the relative importance of $\beta_{\text {stat }}$ of $(b)$ applies, except where the probes carry acidic hydrogens (e.g., nitrophenols and nitroanilines), the latter being "biased" toward solvent basicity [45].

In summary, the Taft-Kamlet-Abboud equation may be expanded to include solvent lipophilicity, as given by $\log P$ (or any equivalent scale). At least for the probes shown in Table $1, \beta_{\mathrm{S}}$ may be dropped, so that solvent polarity is described in terms of its acidity, dipolarity/polarizability, and lipophilicity. Equation 4 is general, unless the molecular structure of the probe makes it biased toward a particular solvent property.

\section{SOLVATION IN BINARY SOLVENT MIXTURE}

Figure 4 shows the dependence of $E_{\mathrm{T}}\left(\mathrm{MePMBr}_{2}\right)$ on $\chi_{\mathrm{W}}$ for mixtures of $\mathrm{W}$ with $\mathrm{MeOH}, 1$-propanol, $\mathrm{PrOH}, \mathrm{MeCN}$, and dimethylsulfoxide (DMSO) at $25{ }^{\circ} \mathrm{C}$ [45]. This dependence is not linear (i.e., it is not ideal); a large body of solvatochromic data shows that this is a general behavior [10,13-16,37-45]. How can this dependence be explained? Answering this question is not straightforward because one has
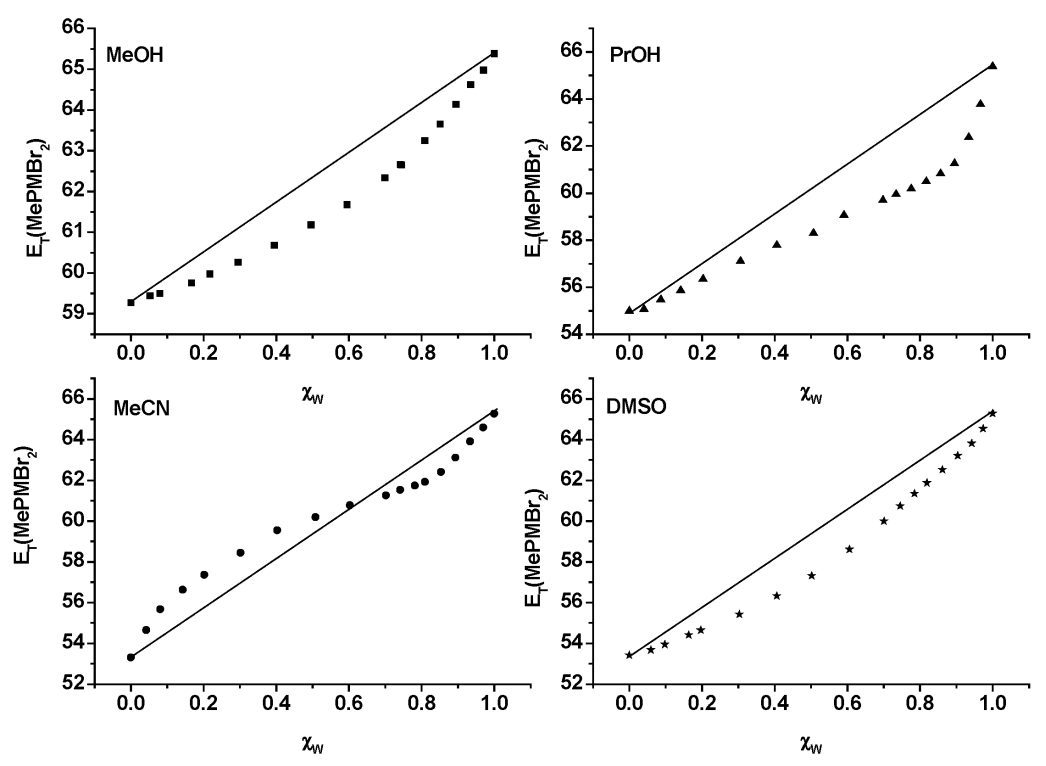

Fig. 4 Dependence of $E_{\mathrm{T}}\left(\mathrm{MePMBr}_{2}\right)$ on $\chi_{\mathrm{W}}$ for mixtures of water with $\mathrm{A}, \mathrm{MeOH} ; \mathrm{B}, \mathrm{PrOH} ; \mathrm{C}, \mathrm{MeCN}$ and D, DMSO, respectively, at $25^{\circ} \mathrm{C}$. 
to consider solvent/solvent interactions, as well as interactions of the probe with each solvent present. With regard to the first point, the dependence of many macroscopic properties (e.g., density, $\varepsilon_{\mathrm{r}}$, etc.) of binary mixtures on their composition is not linear, as shown in Fig. 5. It may be argued that this macroscopic non-ideality is reflected at the molecular level, so that a nonlinear dependence of $E_{\mathrm{T}}$ (probe) on $\chi_{\mathrm{W}}$ is not unexpected.
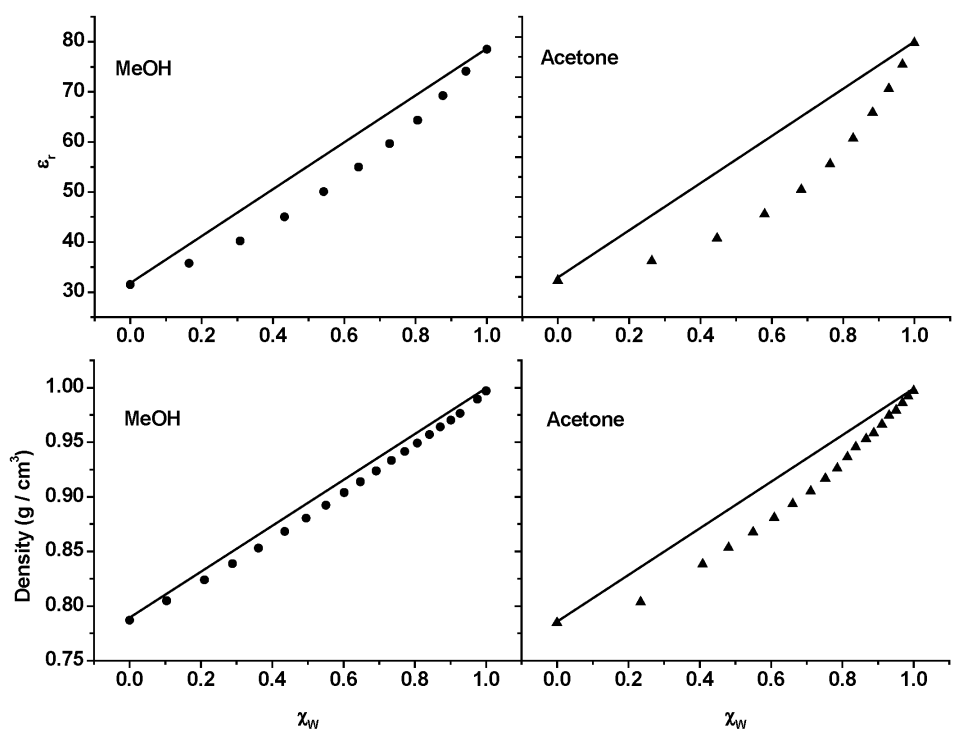

Fig. 5 Dependence of the relative permittivity, $\varepsilon_{\mathrm{r}}$, and density of mixtures of water with methanol or acetone on the mole fraction of water $\chi_{\mathrm{W}}$, at $25{ }^{\circ} \mathrm{C}$ [42b]. The straight lines that connect the points of the two pure solvents represent the ideal behavior.

Considering probe/solvent interactions, the observed deviations from linearity may result from the so-called "preferential solvation" of the probe by one of the components of the mixture. In principle, this phenomenon may include contributions from:

i. "Dielectric enrichment", which denotes enrichment of the probe solvation shell in the solvent of higher $\varepsilon_{\mathrm{r}}$, due to probe dipole/solvent dipole interactions. This interaction, if it occurs, implies $a$ positive deviation in the $E_{\mathrm{T}}$ (probe) vs. $\chi_{\text {higher }}\left(\varepsilon_{\mathrm{r}}\right)$ plot, even when Onsager dielectric function [ $f\left(\varepsilon_{\mathrm{r}}=2\left(\varepsilon_{\mathrm{r}}-1\right) / 2 \varepsilon_{\mathrm{r}}+1\right]$ of the mixture is linear [10]. Indeed, $E_{\mathrm{T}}$ (probe) were found to be nonlinear functions of $\chi_{\text {higher }}\left(\varepsilon_{\mathrm{r}}\right)$ for the ideal binary mixtures cyclohexane-THF and cyclohexane-1-butanol $[37,43]$. This interaction mechanism is nonspecific and is, therefore, independent of probe structure.

ii. Deviation from linearity may be due to probe/solvent specific interactions (e.g., hydrogen-bonding and hydrophobic interactions); these depend on the $\mathrm{p} K_{\mathrm{a}}$ and $\log P$ of both probe and solvent.

iii. Finally, nonlinear behavior results from solvent microheterogeneity, i.e., where one component of the mixed solvent prefers a molecule of the same type. A large body of experimental data and theoretical calculations (e.g., of the Kirkwood-Buff integral functions, which describe W/W, S/S, and S/W interactions) has shown that many binary mixtures are microheterogeneous; there exist microdomains composed of organic solvent surrounded by water, and of water solvated by organic solvent. The onset and composition of these microdomains depend on the pair of solvents. There exists the possibility of solvation of the probe by one of the two solvent microdomains $[10,50,51 \mathrm{a}, \mathrm{b}]$. 
The important consequence of solvation mechanisms i to iii is that the composition of the solvation shell differs from that of bulk mixture; this difference is important because it means that the solvation shell will be enriched in one solvent, say, alcohol or DMSO; this may be advantageously employed in reactions where desolvation is important (e.g., acyl transfers) and/or in reactions where the transition state is much more polar than the reactants (e.g., the Menshutkin reaction) [51c].

The following question now arises: Is it possible to calculate the composition of the probe solvation shell and to compare it with that of bulk mixture? This problem has been solved by calculating the so-called solvent "fractionation factor", $\varphi$, defined as the ratio: (composition in the probe solvation shell)/(composition of bulk mixture). Values of $\varphi$ depend on the position of the exchange equilibria between solvent species in bulk solvent with those in the probe solvation shell; calculation of $\varphi$ went through several refinements, as discussed below:

- The solvent exchange equilibrium considered was that between the two pure solvents in the solvation shell where, for simplicity, we consider that the probe solvatochromic response is affected by the exchange of one solvent molecule [52]:

$$
\operatorname{Probe}(\mathrm{S})+\mathrm{W} \rightleftharpoons \operatorname{Probe}(\mathrm{W})+\mathrm{S}
$$

This approach has been applied to mixtures of $\mathrm{W}$ and alcohols and/or $\mathrm{W}$ and dipolar aprotic solvents. Analysis of the solvatochromic data obtained showed that this model is an oversimplification of a complex situation. For example, in some cases the $E_{\mathrm{T}}$ (probe) vs. $\chi_{\mathrm{W}}$ curve could not be reproduced by using a single $\varphi$ over the whole composition range. Although the problem was solved by dividing $\chi_{\mathrm{W}}$ into two regions (e.g., 0 to 0.5 and 0.5 to 1 ), values of $\varphi$ calculated were puzzling because they indicated that $\mathrm{RB}$, a very hydrophobic probe (solubility in water, ca. $2 \times$ $10^{-6} \mathrm{~mol} / \mathrm{L}$ [13]), is preferentially solvated by water, in the water-poor region! [37].

- Later, the formation of a "mixed" or hydrogen-bonded species, S-W, has been introduced: $\mathrm{W}+\mathrm{S} \rightleftharpoons 2 \mathrm{~S}-\mathrm{W}$. Formation of $\mathrm{S}-\mathrm{W}$ has been limited to the probe solvation shell; the coefficient 2 in the right-hand side of the equilibrium "is necessary to keep the number of solvent molecules constant" [53]. The convenience of this approach is that bulk [S-W] is not considered in the calculations, so that solvent fractionation factors are based on analytical concentrations of $\mathrm{W}$ and $\mathrm{S}$. This model has been employed to fit $E_{\mathrm{T}}$ (probe) vs. $\chi_{\mathrm{W}}$ data [39,53]. Its validity has been questioned, however, based on the following arguments: Deviations of the macroscopic properties of solvent mixtures from ideality (e.g., Fig. 5) have been explained on the bases of formation of $(\mathrm{S}-\mathrm{W})$ complexes in bulk solution; At equilibrium, (S-W) in the probe solvation shell must be in equilibrium with the same species in bulk solvent. Consequently, concentration of (S-W) should be incorporated in the model; if, on the other hand, the formation of (S-W) is restricted to the solvation shell, then the model cannot be employed to describe the ideal case, i.e., where solvation shell composition is equal to that of bulk solvent. The reason is that only one of the solvent "pseudophases", namely that of the solvation shell, contains the species (S-W).

- More recently, we have explicitly considered the formation of 1:1 (S-W) in the entire system (bulk mixture and solvation shell), this led to the following solvent exchange equilibria [40]:

$$
\begin{aligned}
& \mathrm{S}+\mathrm{W} \rightleftharpoons \mathrm{S}-\mathrm{W} \\
& \operatorname{Probe}(\mathrm{S})_{\mathrm{m}}+m(\mathrm{~S}-\mathrm{W}) \rightleftharpoons \operatorname{Probe}(\mathrm{S}-\mathrm{W})_{m}+m \mathrm{~S} \\
& \operatorname{Probe}(\mathrm{W})_{\mathrm{m}}+m(\mathrm{~S}-\mathrm{W}) \rightleftharpoons \operatorname{Probe}(\mathrm{S}-\mathrm{W})_{m}+m \mathrm{~W}
\end{aligned}
$$

These, after algebraic manipulation, lead to the following solvent fractionation factors:

$$
\varphi_{\mathrm{W} / \mathrm{S}}=\frac{x_{\mathrm{W}}^{\text {Probe }} / x_{\mathrm{S}}^{\text {Probe }}}{\left(x_{\mathrm{W}}^{\mathrm{Bk} ; \text { Effective }} / x_{\mathrm{S}}^{\mathrm{Bk} ; \text { Effective }}\right)^{\mathrm{m}}}
$$




$$
\begin{gathered}
\varphi_{\mathrm{S}-\mathrm{W} / \mathrm{S}}=\frac{x_{\mathrm{S}-\mathrm{W}}^{\text {Probe }} / x_{\mathrm{S}}^{\text {Probe }}}{\left(x_{\mathrm{S}-\mathrm{W}}^{\mathrm{Bk} \text { Effective }} / x_{\mathrm{S}}^{\mathrm{Bk} ; \text { Effective }}\right)^{\mathrm{m}}} \\
\varphi_{\mathrm{S}-\mathrm{W} / \mathrm{W}}=\frac{x_{\mathrm{S}-\mathrm{W}}^{\text {Probe }} / x_{\mathrm{W}}^{\text {Probe }}}{\left(x_{\mathrm{S}-\mathrm{W}}^{\mathrm{Bk} ; \text { Effective }} / x_{\mathrm{W}}^{\mathrm{Bk} ; \text { Effective }}\right)^{\mathrm{m}}}
\end{gathered}
$$

where $(m)$ represents the number of solvent molecules whose exchange in the probe solvation shell affects $E_{\mathrm{T}}$ (probe); usually $m \leq 2$, Bk refers to bulk solvent, and the concentration of the solvent species are "effective" not analytical. Note that $(m)$ should not be confused with the total number of solvent molecules that solvate the probe.

Regarding this solvation model, several points deserve comments:

iv. At the outset, it is appropriate to address the use of 1:1 stoichiometry for (S-W), according to eq. 6. This is a practical and convenient assumption because it renders subsequent calculations tractable; it has been extensively employed by others to describe solvatochromism [52,53]. Mixed solvent species with stoichiometry other than 1:1 may be treated, to a good approximation, as mixtures of the 1:1 structure plus excess of a pure solvent. The formation of $(\mathrm{S}-\mathrm{W})$ complexes are manifested by the non-ideal, i.e., nonlinear relationships between compositions and physicochemical properties of binary solvent mixtures, including their densities; dielectric constants; NMR relaxation times; dielectric relaxations; and fluorescence lifetimes of dissolved probes [54]. The Kirkwood-Buff integral functions [51a,b], and electron-impact mass spectroscopy support the formation of (S-W) complexes [55]. Additionally, the 1:1 model has been successfully employed to fit the data of spectroscopic techniques that are particularly suitable to determine the stoichiometry of (S-W) aggregates, including the dependence of the ${ }^{1} \mathrm{H}$ chemical shift (NMR) and/or the peak area and frequency of $\mathrm{v}_{\mathrm{OH}}$ (Fourier transform infrared, FTIR) on concentration of water [56]. Using ${ }^{1} \mathrm{H}$ NMR spectroscopy, the stoichiometry of mixtures of dipolar aprotic solvents and water (in $\mathrm{CCl}_{4}$ ) has been calculated; both 1:1 and 2:1 S-W complexes were considered. The ratios $K_{1-1} / K_{2-1}$ ranged from $26(\mathrm{MeCN}$ ) to 132 (DMSO) [57]. In summary, solvatochromic data can be conveniently analyzed by considering 1:1 ROH-W complexes only.

v. Values of the appropriate fractionation factors are calculated, by iteration, from the dependence of the observed polarity of the binary mixture, $E_{\mathrm{T}}^{\mathrm{obs}}$, on its composition, as shown by eq. 12 :

$$
E_{\mathrm{T}}^{\mathrm{obs}}=\frac{\left(\chi_{\mathrm{S}}^{\mathrm{Bk} \text { Effective }}\right)^{\mathrm{m}} E_{\mathrm{T}}^{\mathrm{S}}+\varphi_{\mathrm{W} / \mathrm{S}}\left(\chi_{\mathrm{W}}^{\mathrm{Bk} ; \text { Effective }}\right)^{\mathrm{m}} E_{\mathrm{T}}^{\mathrm{W}}+\varphi_{\mathrm{S}-\mathrm{W} / \mathrm{S}}\left(\chi_{\mathrm{S}-\mathrm{W}}^{\mathrm{Bk} \text { Effective }}\right)^{\mathrm{m}} E_{\mathrm{T}}^{\mathrm{S}-\mathrm{W}}}{\left(\chi_{\mathrm{S}}^{\mathrm{Bk} ; \text { Effective }}\right)^{\mathrm{m}}+\varphi_{\mathrm{W} / \mathrm{S}}\left(\chi_{\mathrm{W}}^{\mathrm{Bk} \text { Effective }}\right)^{\mathrm{m}}+\varphi_{\mathrm{S}-\mathrm{W} / \mathrm{S}}\left(\chi_{\mathrm{S}-\mathrm{W}}^{\mathrm{Bk} \text { Effective }}\right)^{\mathrm{m}}}
$$

vi. Calculation of $[\mathrm{S}-\mathrm{W}]$ requires knowledge of the dissociation constant, $K_{\text {dissoc }}$, of the hydrogenbonded solvent $\left(1 / K_{\text {dissoc }}=\right.$ the association constant $)$; this has been calculated from the dependence of binary mixture density on its composition. The input data to calculate $K_{\text {dissoc }}$ include densities of the mixtures, the molar masses and volumes of the two components $\left(V_{\mathrm{S}}\right.$ and $V_{\mathrm{W}}$, respectively), along with initial estimates of $K_{\text {dissoc }}$ and $V_{\mathrm{S}-\mathrm{W}}$. That is, $K_{\text {dissoc }}$ and $V_{\mathrm{S} \text {-W }}$ are calculated simultaneously from the same set of experimental data [40-43]. This calculation, however, may be subject to uncertainty because both parameters $\left(K_{\text {dissoc }}\right.$ and $\left.V_{\mathrm{S}-\mathrm{W}}\right)$ are correlated. Recently, $V_{\mathrm{S}-\mathrm{W}}$ was obtained by ab initio calculations, followed by corrections for the temperature and the non-ideality of the binary mixture. The volumes thus calculated were employed as fixed, not adjustable parameters in the calculations of $K_{\text {dissoc }}$ [44]. The effective concentrations 
of the solvent species present are then calculated from their analytical counterparts and $K_{\text {dissoc }}$, as illustrated in Fig. 6 [42b]:

vii. It is clear from eqs. 9-11 that preferential solvation can be readily deduced from the value of $\varphi$. For example, for $\varphi_{\mathrm{W} / \mathrm{S}}>1$, the solvation shell is richer in $(\mathrm{W})$ than the bulk mixture; the converse holds for $\varphi_{\mathrm{W} / \mathrm{S}}<1$, i.e., the probe is preferentially solvated by (S). Finally, a solvent fractionation factor of unity indicates an ideal behavior, i.e., solvation shell and bulk mixture have equal compositions. The same line of reasoning applies to $\varphi_{\mathrm{S}-\mathrm{W} / \mathrm{S}}$ (complex solvent substituting $\mathrm{S}$ ) and $\varphi_{\mathrm{S}-\mathrm{W} / \mathrm{W}}($ complex solvent substituting $\mathrm{W}$ ), eqs. 10 and 11 , respectively;

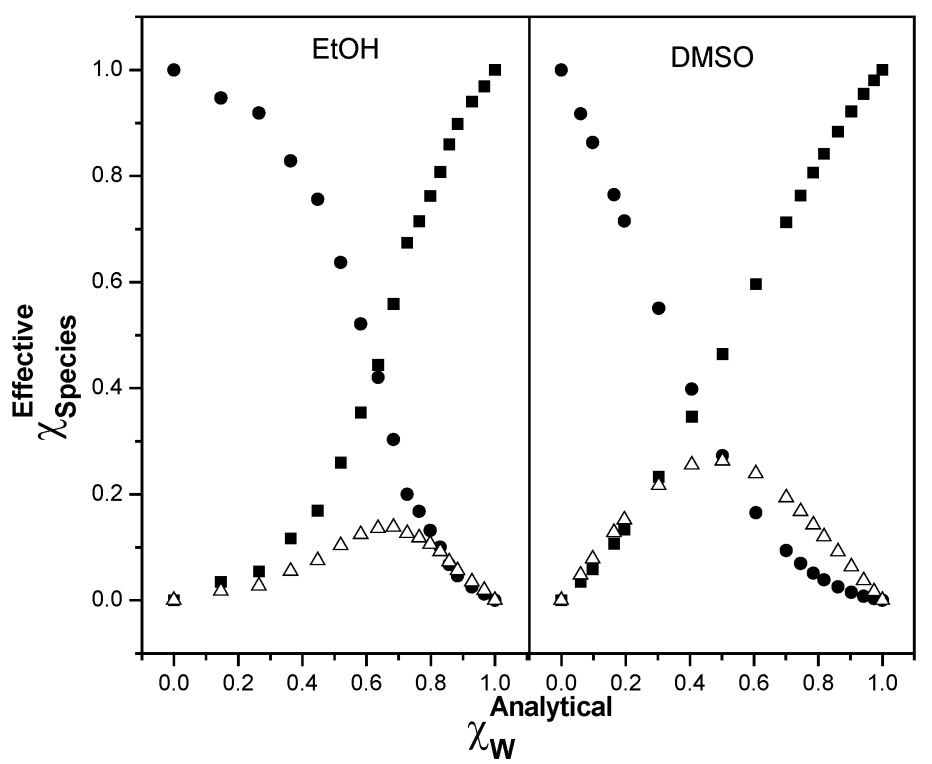

Fig. 6 Dependence of solvent species concentration on $\chi_{\mathrm{W}}$, at $25^{\circ} \mathrm{C}$, for mixture of water with MeOH or DMSO, respectively. The symbols are $(\bullet),(\square)$ and $(\triangle)$ for $(S),(W)$, and $(S-W)$, respectively.

Figure 7 shows typical examples of thermo-solvatochromic data, namely, solvent polarity/temperature/solvent composition contours for $\mathrm{MePMBr}_{2}$ in $\mathrm{MeOH}, \mathrm{PrOH}, \mathrm{MeCN}$, and DMSO, respectively, whereas the corresponding fractionation factors are collected in Table 2 [45]: 

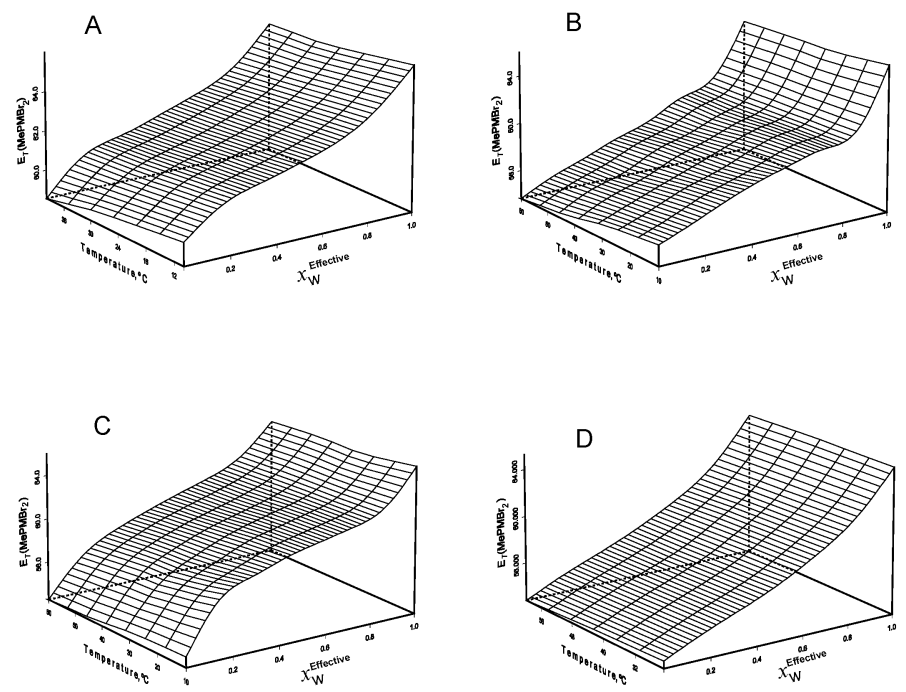

Fig. 7 Solvent polarity/temperature/solvent composition contours for $\mathrm{MePMBr}_{2}$ in $\mathrm{A}, \mathrm{MeOH}$; $\mathrm{B}, \mathrm{PrOH} ; \mathrm{C}, \mathrm{MeCN}$ and D, DMSO.

Table 2 Analysis of thermo-solvatochromic responses of $\mathrm{MePMBr}_{2}$, in $\mathrm{S}-\mathrm{W}$ mixtures, according to eqs. 9-11. ${ }^{\mathrm{a}}$

\begin{tabular}{lcccccccccc}
\hline $\begin{array}{l}\text { Organic } \\
\text { solvent }\end{array}$ & $T /{ }^{\circ} \mathrm{C}$ & $m$ & $\varphi_{\mathrm{W} / \mathrm{S}}$ & $\varphi_{\mathrm{S}-\mathrm{W} / \mathrm{S}}$ & $\varphi_{\mathrm{S}-\mathrm{W} / \mathrm{W}}$ & $E_{\mathrm{T}}(\text { probe })_{\mathrm{S}}$ & $E_{\mathrm{T}}(\text { probe })_{\mathrm{W}}$ & $E_{\mathrm{T}}(\text { probe })_{\mathrm{S}-\mathrm{W}}$ & $r^{2}$ & $\mathrm{Chi}^{2}$ \\
\hline MeOH & 10 & 0.971 & 0.567 & 1.867 & 3.293 & $59.77( \pm 0.04)$ & $65.96( \pm 0.04)$ & $61.32( \pm 0.2)$ & 0.9996 & 0.0022 \\
& 25 & 1.007 & 0.578 & 1.805 & 3.123 & $59.27( \pm 0.03)$ & $65.27( \pm 0.02)$ & $60.99( \pm 0.03)$ & 0.9999 & 0.0008 \\
& 40 & 0.870 & 0.610 & 1.756 & 2.879 & $58.55( \pm 0.04)$ & $65.17( \pm 0.03)$ & $60.67( \pm 0.21)$ & 0.9998 & 0.0015 \\
$\mathrm{PrOH}$ & 10 & 1.580 & 0.211 & 71.138 & 337.147 & $55.45( \pm 0.06)$ & $66.00( \pm 0.08)$ & $59.76( \pm 0.11)$ & 0.9995 & 0.0069 \\
& 25 & 1.359 & 0.215 & 32.546 & 151.377 & $54.95( \pm 0.08)$ & $65.42( \pm 0.11)$ & $59.68( \pm 0.25)$ & 0.9990 & 0.0133 \\
& 40 & 1.300 & 0.233 & 27.653 & 118.682 & $54.34( \pm 0.09)$ & $65.21( \pm 0.12)$ & $59.35( \pm 0.33)$ & 0.9990 & 0.0142 \\
& 60 & 1.110 & 0.239 & 13.105 & 54.833 & $53.70( \pm 0.10)$ & $64.88( \pm 0.13)$ & $59.60( \pm 0.70)$ & 0.9989 & 0.0166 \\
MeCN & 10 & 0.994 & 1.461 & 27.076 & 18.533 & $53.52( \pm 0.08)$ & $65.99( \pm 0.08)$ & $60.14( \pm 0.29)$ & 0.9995 & 0.0075 \\
& 25 & 1.057 & 1.494 & 26.781 & 17.926 & $53.39( \pm 0.14)$ & $65.36( \pm 0.13)$ & $59.58( \pm 0.69)$ & 0.9988 & 0.0199 \\
& 40 & 1.015 & 1.527 & 22.579 & 14.787 & $52.93( \pm 0.06)$ & $65.21( \pm 0.06)$ & $58.90( \pm 0.42)$ & 0.9998 & 0.0043 \\
& 60 & 0.978 & 1.578 & 16.844 & 10.674 & $52.53( \pm 0.04)$ & $64.85( \pm 0.04)$ & $57.67( \pm 0.46)$ & 0.9999 & 0.0018 \\
DMSO & 25 & 0.768 & 0.342 & 0.356 & 1.041 & $53.33( \pm 0.05)$ & $65.25( \pm 0.06)$ & $57.14( \pm 7.00)$ & 0.9999 & 0.0036 \\
& 40 & 0.745 & 0.412 & 0.342 & 0.830 & $53.12( \pm 0.03)$ & $65.13( \pm 0.06)$ & $54.05( \pm 6.70)$ & 0.9998 & 0.0044 \\
& 60 & 0.703 & 0.421 & 0.242 & 0.575 & $52.74( \pm 0.06)$ & $64.81( \pm 0.06)$ & $52.93( \pm 8.25)$ & 0.9998 & 0.0045 \\
& 60 & 0.756 & 0.400 & 0.348 & 0.870 & $52.46( \pm 0.06)$ & $64.43( \pm 0.06)$ & $52.60( \pm 6.00)$ & 0.9998 & 0.0043 \\
\hline
\end{tabular}

From these data, the following can be deduced:

viii. The goodness of fit of the model to thermo-solvatochromic data is shown by values of $\left(\mathrm{r}^{2}\right)$ and $\mathrm{Chi}^{2}$, and by the excellent agreement between experimental and calculated $E_{\mathrm{T}}$ (probe) $)_{\mathrm{S}}$, and $E_{\mathrm{T}}$ (probe) ${ }_{\mathrm{W}}$, respectively. Values of $(m)$ are close to unity, and generally decrease as a function of increasing $T$. Likewise, all values of $\varphi, E_{\mathrm{T}}$ (probe $)_{\mathrm{S}}$ and $E_{\mathrm{T}}(\text { probe })_{\mathrm{W}}$ decrease as a function of increasing $T$. This probe desolvation agrees with the known effect of temperature on solvent structure, due to less efficient hydrogen-bonding and dipolar interactions [51]. 
ix. Solvation by aqueous alcohols, $\mathrm{ROH}-\mathrm{W}$, is considered first. Values of $\varphi_{\mathrm{W} / \mathrm{ROH}}$ are $<$ unity, i.e., water is not efficient in displacing the alcohol from the probe solvation shell. Whereas water and alcohols may solvate the probe by hydrogen bonding to its phenolate oxygen, $\mathrm{ROH}$ may further interact by hydrophobic interactions. The importance of the latter has been discussed above for pure solvents (eq. 4) and may be further corroborated by the fact that the order observed is $\varphi_{\mathrm{W} / \mathrm{MeOH}}>\varphi_{\mathrm{W} / \mathrm{PrOH}}$, in the temperature range investigated. That is, water displaces $\mathrm{MeOH}$, a stronger acid $\left(\mathrm{p} K_{\mathrm{a}}=15.5\right)$, but less hydrophobic solvent $(\log P=-0.77)$, more efficiently than PrOH, a weaker acid $\left(\mathrm{p} K_{\mathrm{a}}=16.1\right)$, but more hydrophobic solvent $(\log P=0.25)$ [58]. All $\varphi_{\mathrm{ROH}-\mathrm{W} / \mathrm{ROH}}$ and $\varphi_{\mathrm{ROH}-\mathrm{W} / \mathrm{W}}$ are $>1$, indicating that the probes are preferentially solvated by $\mathrm{ROH}-\mathrm{W}$; all $\varphi_{\mathrm{ROH}-\mathrm{W} / \mathrm{W}}$ are $>$ the corresponding $\varphi_{\mathrm{ROH}-\mathrm{W} / \mathrm{ROH}}$, indicating that (ROH-W) displaces (W) more efficiently than $\mathrm{ROH}$. The efficiency of (ROH-W) in displacing alcohol and/or water from the solvation shells does not seem to be due to a better hydrogen-bonding ability than those of the precursor solvents; it is due to (the additional) hydrophobic interactions, as argued above.

x. For mixtures of water with dipolar aprotic solvents $\varphi_{\mathrm{W} / \mathrm{MeCN}}>1$, whereas $\varphi_{\mathrm{W} / \mathrm{DMSO}}<1$, i.e., water is more efficient in displacing MeCN than DMSO from the solvation shell. MeCN can solvate positive centers better than negative ones, i.e., it interacts less with the probe phenolate oxygen, being displaced by water, because the latter is capable of solvating anions and cations effectively [59]. Solvation by aqueous DMSO merits a comment: Whereas $\varphi_{\mathrm{W} / D M S O}$ is $<1$, i.e., similar to solvation by alcohols, values of $\varphi_{\text {DMSO-W/W }}$ and $\varphi_{\text {DMSO-W/DMSO }}$ are less than, or close to unity. This is the first example of (S-W) species that is inefficient in displacing its precursor components from the probe solvation shell. Consider first the exchange of the pure solvents. Values of $\varphi_{\mathrm{W} / \mathrm{DMSO}}$ are $<1$ probably because the organic solvent may solvate the probe by strong dipole/dipole and hydrophobic interactions, akin to those operative in aqueous DMSO [60] The small magnitudes of $\varphi_{\text {DMSO-W/W }}$ and $\varphi_{\text {DMSO-W/DMSO }}$ can be attributed to the fact that the interaction of DMSO with $\mathrm{W}$ attenuates the solvation efficiency of the complex solvent. There is ample evidence that DMSO/W interactions are stronger than W/W interactions [61-64]. The molecular structure of the mixed solvent is given by: $\left(\mathrm{CH}_{3}\right)_{2} \mathrm{~S}^{\delta+}=\mathrm{O}^{\delta-} \ldots \mathrm{Ha}^{\delta+}-\mathrm{O}^{\delta-}-H_{\mathrm{b}}$, where $H_{\mathrm{b}}$ is the site for hydrogen bonding with the probe phenolate oxygen. As argued elsewhere, the formation of mixed solvent partially deactivates $H_{\mathrm{b}}$ toward further hydrogen bonding, this deactivation is greater the stronger the basicity of S [65]. Because of the high basicity of DMSO, its mixed solvent (with $\mathrm{W}$ ) may be considered as a deactivated species both in hydrogen bonding to the probe phenolate oxygen, and electrostatic interaction with the probe positively charged nitrogen, this leads to the small $\varphi$ observed;

xi. Table 2 shows that as a function of increasing temperature, $(m), E_{\mathrm{T}}(\text { probe })_{\mathrm{S}}, E_{\mathrm{T}}(\text { probe })_{\mathrm{W}}, \varphi_{\mathrm{S}-\mathrm{W} / \mathrm{S}}$ and $\varphi_{\mathrm{S}-\mathrm{W} / \mathrm{W}}$ decrease, whereas $\varphi_{\mathrm{W} / \mathrm{S}}$ increases. The decrease in polarities of pure solvents can be attributed to a decrease of solvent stabilization of the probe ground state, as a result of the concomitant decrease of solvent structure, and hydrogen-bonding ability [54b,66]. Preferential "clustering" of water and solvents as a function of increasing temperature means that the strength of $(\mathrm{S}-\mathrm{W})$ interactions decrease in the same direction, with a concomitant decrease in its ability to displace both water and solvent $[50,51,55 \mathrm{a}, 55 \mathrm{~b}]$. This explains the decrease of $\varphi_{\mathrm{S}-\mathrm{W} / \mathrm{S}}$ and $\varphi_{\mathrm{S}-\mathrm{W} / \mathrm{W}}$ as a function of increasing T. It is known that the structure of water is less affected by temperature increase, relative to most organic solvents [51]. Consequently, hydrogen bonding of water with the probe ground state is less susceptible to temperature increase than that of the organic component. This leads to a measurable "depletion" of the organic solvent in the probe solvation coordination shell, so that $\varphi_{\mathrm{W} / \mathrm{S}}$ increases as a function of increasing temperature. Table 2 is for a single probe. Data for other $\mathrm{RPMBr}_{2}, \mathrm{R}=n$-butyl and $n$-octyl (not shown) have indicated that solvation in the same group of pure solvents, and in the same binary solvent mixture is sensitive to the lipophilicity of the probe [45]. 


\section{CONCLUSIONS}

Studies of solvatochromism and thermo-solvatochromism are important because $E_{\mathrm{T}}$ (probe) quantifies the relative importance to solvation of the physicochemical properties of both substrate and solvent, or mixtures of solvents. This subject is generating much interest because of the intense recent research on "green" solvents. It is hoped that this short review contributes to increasing the awareness of chemists to its conclusion: In binary solvent mixtures, the composition of the solvation shells of the species of interest (reagents and/or activated complexes) differ from that of bulk medium. This difference may be exploited, e.g., in $\mathrm{S}_{\mathrm{N}} 2$ and acyl transfers, where desolvation of the attacking nucleophile enhances the reaction rate.

Another important aspect is the relevance of these studies to chemical kinetics. Although desolvation of reactants and activated complexes is known to contribute to temperature effects on reaction rates, there is no simple way to calculate its contribution to $\Delta H^{\neq}$from the Arrhenius plot. This desolvation energy is readily calculated from thermo-solvatochromism. Our results have shown that the magnitude of this energy is sizeable (e.g., range from 2.1 to $3.7 \mathrm{kcal} \mathrm{mol}^{-1}$ over a $50{ }^{\circ} \mathrm{C}$ range for $\mathrm{WB}$ in aqueous alcohols) relative to the activation enthalpies of many organic reactions [42].

Another point worth mentioning is the relevance of solvatochromic data to the above-discussed complex dependence of $k_{\mathrm{obs}}$ on medium composition, for reactions carried out in binary mixtures. Provided that the phenomenon involved in solvatochromism (excitation from the ground to the first excited state) serves as a model for reactions where there is a large difference in dipolarity between the reactants and the corresponding activated complexes, one can draw a parallel between the responses of the probe and of the reaction to the microheterogeneity of the medium. An example of this use is shown in Fig. 8, for the $\mathrm{pH}$-independent hydrolysis of two activated esters, namely, 4-nitrophenyl chloroformate (NPCF) and 4-nitrophenyl heptafluorobutyrate (NPFB), respectively, in aqueous MeCN [32]. The similar responses to solvent composition are remarkable because of their distinct origins, an acyl transfer reaction, and excitation of a ground state, respectively.
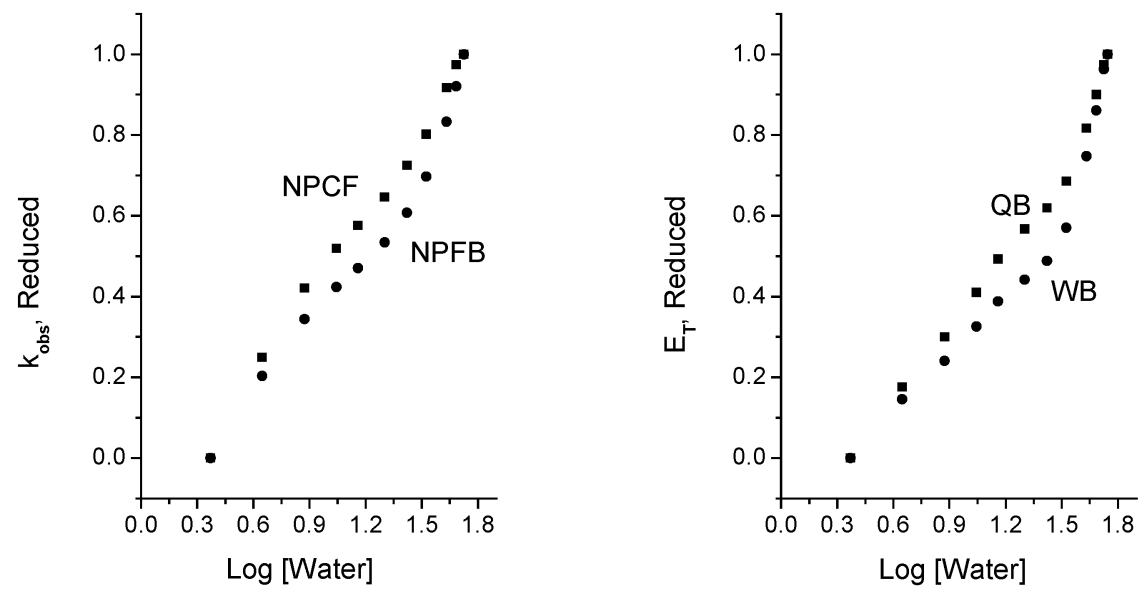

Fig. 8 Plots of the dependence of $\log \left(k_{\text {obs }}\right)$ and of $E_{\mathrm{T}}$ (probe) on $\log [\mathrm{W}]$, at $25^{\circ} \mathrm{C}$. Reduced $\log \left(k_{\text {obs }}\right)$ and reduced $E_{\mathrm{T}}$ (probe) are employed, so that results from different phenomena (ester hydrolysis and probe excitation, respectively) may be directly compared. Values of $E_{\mathrm{T}}$ (probe) in pure water and pure MeCN are, respectively, 64.62 and $52.97 \mathrm{kcal} / \mathrm{mol}(\mathrm{QB})$ and 70.24 and $54.72 \mathrm{kcal} / \mathrm{mol}(\mathrm{WB})$. 


\section{ACKNOWLEDGMENTS}

I would like to thank FAPESP (State of São Paulo Research Foundation) for financial support; CNPq (National Council for Scientific and Technological Research) for a research productivity fellowship; my research coworkers L. P. Novaki, E. B. Tada, P. L. Silva, M. Antonious, C. T. Martins, M. S. Lima, and E. L. Bastos for doing the work reported here; and P. A. R. Pires and C. Guizzo for their help.

\section{REFERENCES}

1. P. T. Anastas, J. C. Warner. Green Chemistry: Theory and Practice, p. 30, Oxford University Press, New York (1998).

2. P. Tundo, P. T. Anastas, D. S. Black, J. Breen, T. Collins, S. Memoli, J. Miyamoto, M. Polyakoff, W. Tumas. Pure Appl. Chem. 72, 1207 (2000).

3. P. T. Anastas, J. B. Zimmerman. Environ. Sci. Technol. 37, 95A (2003).

4. W. Leinter. Acc. Chem. Res. 35, 746 (2002).

5. T. Welton. Chem. Rev. 99, 2071 (1999).

6. P. Wassescheid, W. Keim. Angew. Chem., Int. Ed. 39, 3772 (2000).

7. J. Dupont, R. F. de Souza, P. A. Z. Suarez. Chem. Rev. 102, 3667 (2002).

8. O. Pytela. Collect. Czech. Chem. Commun. 53, 1333 (1988).

9. P. Politzer, J. S. Murray. Quantitative Treatments of Solute-Solvents Interactions, p. 230, Elsevier, New York (1994).

10. P. Suppan, N. Ghoneim. Solvatochromism, p. 21, The Royal Society of Chemistry: Cambridge (1997).

11. J.-L. M. Abboud, R. Notario. Pure Appl. Chem. 71, 645 (1999).

12. G. Hefter, Y. Marcus, W. E. Waghorne. Chem. Rev. 102, 2773 (2002).

13. C. Reichardt. Solvents and Solvent Effects in Organic Chemistry, $3^{\text {rd }}$ ed., pp. 5, 329, 389, VCH, Weinheim (2003).

14. C. Reichardt. Pure Appl. Chem. 76, 1903 (2004).

15. C. Reichardt. Green Chem. 7, 339 (2005).

16. A. R. Katritzky, D. C. Fara, H. Yang, K. Taemm, T. Tamm, M. Karelson. Chem. Rev. 104, 175 (2004).

17. M. K. Chantooni Jr., I. M. Kolthoff. J. Phys. Chem. 79, 1176 (1975).

18. F. Nome, S. A. Chang, J. H. Fendler. J. Colloid Interface Sci. 56, 146 (1976).

19. M. T. Rogers, J. L. Burdett. J. Am. Chem. Soc. 86, 2105 (1964).

20. H. D. Zook, T. J. Russo. J. Am. Chem. Soc. 82, 1258 (1960).

21. (a) S. Winstein, A. H. Fainberg. J. Am. Chem. Soc. 78, 2770 (1956); (b) S. Winstein, A. H. Fainberg. J. Am. Chem. Soc. 79, 5937 (1957).

22. (a) R. E. Swart, L. J. LeRoux. J. Chem. Soc. 2110 (1956); (b) R. E. Swart, L. J. LeRoux. J. Chem. Soc. 406 (1957).

23. N. J. Buurma, A. M. Herranz, J. B. F. N. Engberts. J. Chem. Soc., Perkin Trans. 2113 (1999).

24. (a) D. S. Kemp, K. G. Paul. J. Am. Chem. Soc. 97, 7305 (1975); (b) J. W. Grate, R. A. Mcgill, D. Hilvert. J. Am. Chem. Soc. 115, 8577 (1993).

25. J. B. F. N. Engberts. Pure Appl. Chem. 67, 823 (1995).

26. M. J. S. Dewar, D. M. Storch. J. Chem. Soc., Perkin Trans. 2877 (1989).

27. E. Humeres, R. J. Nunes, V. G. Machado, M. D. D. Gasques, C. Machado. J. Org. Chem. 66, 1163 (2001).

28. O. S. Sørensen. Acta Chem. Scand. A 30, 673 (1976).

29. O. A. El Seoud, M. I. El Seoud, J. P. S. Farah. J. Org. Chem. 62, 5928 (1997).

30. J. W. Henederson, P. Haake. J. Org. Chem. 42, 3989 (1977).

31. J. W. Wijnen, S. Zavarise, J. B. F. N. Engberts. J. Org. Chem. 61, 2001 (1996). 
32. O. A. El Seoud, F. Siviero. J. Phys. Org. Chem. 19, 793 (2006).

33. M. H. Abraham, P. L. Grellier. J. Chem. Soc., Perkin Trans. 21735 (1976).

34. (a) M. J. Kamlet, R. W. Taft. Prog. Phys. Org. Chem. 13, 485 (1981); (b) M. H. Abraham, P. L. Grellier, J.-L. M Abboud, R. M. Doherty, R. W. Taft. Can. J. Chem. 66, 2673 (1988); (c) C. Laurence, P. Nicolet, M. T. Dalati, J.-L. M Abboud, R. Notario. J. Phys. Chem. 98, 5807 (1994)

35. A. J. Leo, C. Hansch. Perspect. Drug Discov. Des. 17, 1 (1999).

36. L. P. Novaki, O. A. El Seoud. Ber. Bunsen-Ges. Phys. Chem. 100, 648 (1996).

37. (a) L. P. Novaki, O. A. El Seoud. Ber. Bunsen-Ges. Phys. Chem. 101, 105 (1997); (b) L. P. Novaki, O. A. El Seoud. Ber. Bunsen-Ges. Phys. Chem. 101, 902 (1997).

38. E. B. Tada, L. P. Novaki, O. A. El Seoud. J. Phys. Org. Chem. 13, 679 (2000).

39. M. S. Antonious, E. B. Tada, O. A. El Seoud. J. Phys. Org. Chem. 15, 403 (2002).

40. E. B. Tada, P. L. Silva, O. A. El Seoud. J. Phys. Org. Chem. 16, 691 (2003).

41. E. B. Tada, P. L. Silva, O. A. El Seoud. Phys. Chem. Chem. Phys. 5, 5378 (2003).

42. (a) E. B. Tada, P. L. Silva, C. Tavares, O. A. El Seoud. J. Phys. Org. Chem. 18, 398 (2005); (b) E. B. Tada. Ph.D. thesis, University of São Paulo (2004).

43. C. T. Martins, M. S. Lima, O. A. El Seoud. J. Phys. Org. Chem. 18, 1072 (2005).

44. E. L. Bastos, P. L. Silva, O. A. El Seoud. J. Phys. Chem. A 110, 10287 (2006).

45. C. T. Martins, M. S. Lima, O. A. El Seoud. J. Org. Chem. 71, 9068 (2006).

46. T. Hill, P. Lewicki. Statistic Methods and Applications, A Comprehensive Reference for Science, Industry and Data Mining, $1^{\text {st }}$ ed., p. 555, Statsoft, Tulsa (2006).

47. L. P. Novaki, O. A. El Seoud. Phys. Chem. Chem. Phys. 1, 1957 (1999).

48. O. A. El Seoud. J. Mol. Liq. 72, 85 (1997).

49. W. Taft, M. H. Abraham, R. M. Doherty, M. J. Chalet. J. Am. Chem. Soc. 107, 3105 (1985).

50. (a) I. Shulgin, E. Ruckenstein. J. Phys. Chem. B 103, 872 (1999); (b) I. Shulgin, E. Ruckenstein. J. Phys. Chem. B 103, 2496 (1999).

51. (a) Y. Marcus. Chem. Soc. Rev. 22, 409 (1993); (b) Y. Marcus. Monatsh. Chem. 132, 1387 (2001); (c) J.-L. Abboud, A. Douhal, M. J. Arín, M. T. Diez, H. Homan, G. Guihéneuf. J. Phys. Chem. 93, 214 (1989).

52. (a) E. Bosch, M. Rosés. J. Chem. Soc., Faraday Trans. 88, 3541 (1992); (b) M. Rosés, C. Ràfols, J. Ortega, E. Bosch. J. Chem. Soc., Perkin Trans. 21607 (1995).

53. (a) C. Ràfols, M. Rosés, E. Bosch. J. Chem. Soc., Perkin Trans. 2243 (1997); (b) U. Buhvestov, F. Rived, C. Ràfols, E. Bosch, M. Rosés. J. Phys. Org. Chem. 11, 185 (1998).

54. (a) G. Roux, D. Roberts, G. Perron, J. E. Desnoyers. J. Solution Chem. 9, 629 (1980); (b) R. Zana, M. J. Eliebari. J. Phys. Chem. 97, 11134 (1993); (c) A. Sacco, F. M. De Cillis, M. Holz. J. Chem. Soc., Faraday Trans. 94, 2089 (1998); (d) K. R. Harris, P. J. Newitt. J. Phys. Chem. A 103, 6508 (1999); (e) P. Petong, R. Pottel, U. Kaatze. J. Phys. Chem. A 104, 7420 (2000).

55. (a) K. Nishikawa, H. Hayashi, T. Iijima. J. Phys. Chem. 93, 6559 (1989); (b) M. Huelsekopf, R. Ludwig. J. Mol. Liq. 85, 105 (2000).

56. (a) J.-S. Chen, J.-C. Shiao. J. Chem. Soc., Faraday Trans. 90, 429 (1994); (b) F. Eblinger, H.-J. Schneider. J. Phys. Chem. 100, 5533 (1996); (c) J. J. Max, S. Daneault, C. Chapados. Can. J. Chem. 80, 113 (2002).

57. J. F. Coetzee, A. Hussam. J. Solution Chem. 11, 395 (1982).

58. G. B. Barlin, D. D. Perrin. Quart. Rev. 20, 75 (1966).

59. G. Gopalakrishnan, J. L. Hogg. J. Org. Chem. 49, 3161 (1984).

60. D. N. Shin, J. W. Wijnen, J. B. F. N. Engberts, A. Wakisaka. J. Phys. Chem. B 105, 6759 (2001).

61. I. A. Borin, M. S. Skaf. J. Chem. Phys. 110, 6412 (1999).

62. K. Mizuno, S. Imafuji, T. Ochi, T. Ohta, S. Maeda. J. Phys. Chem. B 104, 11001 (2000).

63. S. N. Shashkov, M. A. Kiselev, S. N. Tioutiounnikov, A. M. Kiselev, P. Lesieur. Physica B 271, 184 (1999). 
64. J. T. Cabral, A. Luzar, J. Teixeira, M. C. Bellissent-Funel. J. Chem. Phys. 113, 8736 (2000).

65. (a) B. Kingston, M. C. R. Symons. J. Chem. Soc., Faraday Trans. 2 69, 978 (1973); (b) M. C. R. Symons. Pure Appl. Chem. 58, 1121 (1986).

66. J. R. Haak, J. B. F. N. Engberts. Recl. Trav. Chim. 105, 307 (1986). 